\title{
Control Automático de Voltaje en Sistemas Eléctricos de Potencia basado en Sistemas Expertos
}

\author{
Jorge C. Vaschetti ${ }^{(1)}$, Fernando Magnago ${ }^{(2)}$ y Víctor H. Sauchelli( ${ }^{(3)}$ \\ (1) GECaP, Facultad Regional Córdoba, Universidad Tecnológica Nacional, Maestro M. Lopez \\ esq. Cruz Roja Argentina, Ciudad Universitaria, X5016ZAA Córdoba-Argentina \\ (e-mail: jvaschetti@scdt.frc.utn.edu.ar). \\ (2) GASEP, Facultad de Ingeniería, Universidad Nacional de Río Cuarto, Ruta Nac. 36 Km. 601, \\ X5804BYA Río Cuarto, Córdoba-Argentina. \\ (3) LIMAC, Facultad de Ciencias Exactas Físicas y Naturales, Universidad Nacional de Córdoba, \\ X5000JJC Córdoba-Argentina.
}

Recibido Mar. 06, 2012; Aceptado Abr. 26, 2012; Versión final recibida May. 06, 2012

\begin{abstract}
Resumen
En este trabajo se presenta el diseño de un controlador experto para el nivel de voltaje en un sistema eléctrico de potencia. La utilización del concepto de nodo piloto permite mantener la lista de controladores estable ante los cambios topológicos de la red. La normativa del Operador Eléctrico de la Provincia de Alberta - Canadá, es utilizada para lograr el perfil óptimo de tensiones. La validación del controlador se realiza mediante una simulación sobre un caso de biblioteca y se compara la solución obtenida con la brindada por un flujo óptimo de potencia y con la de un algoritmo genético. Como resultado fundamental se obtiene el tratamiento unificado del perfil de tensiones y el despacho óptimo de la potencia reactiva sin necesidad de recurrir a un algoritmo de dos pasos. El controlador propuesto resulta apto para su aplicación en línea y en tiempo real, así como flexible a la hora de tratar con problemas de difícil formulación analítica.
\end{abstract}

Palabras clave: Sistema Experto, Control Secundario de Voltaje, Flujo Optimo de Potencia, Sistema de Potencia, CLIPS, MATLAB.

\section{Automatic Voltage Control in Electrical Power System based on Expert Systems.}

\begin{abstract}
This paper presents the design of an expert controller applied to voltage level control in a power system. The use of pilot node concept allows to keep stable the list of controllers against topological changes in the network. To achieve the optimal voltage profile, the Alberta Electric System Operator regulations are used. The controller validation is performed by simulating a case library and the solution is compared with results provided by an optimal power flow and a genetic based algorithm. The main advantage of the propose model is the unified treatment of the voltage profile and the reactive dispatch without the need of a two-step algorithm. The proposed controller is suitable for online real-time applications and it is flexible when dealing with problems that present a complex analytical formulation.
\end{abstract}

Keywords: Expert System, Secondary Voltage Control, Optimal Power Flow, Power System, CLIPS, MATLAB. 


\section{INTRODUCCIÓN}

Un adecuado perfil de tensiones es necesario para mantener el sistema eléctrico de potencia operando en forma segura, existen una serie de eventos que pueden afectar este perfil, conexiones y desconexiones no programadas de cargas, de generación, de líneas de transmisión, operación incorrecta, o cualquier otro dispositivo que pueda salir o entrar en servicio en forma programada o no; el elemento de control para mitigar estas contingencias es la potencia reactiva, la cual es provista básicamente por el sistema de generación (Kundur, 1994).

Un inadecuado monitoreo y control de voltaje pueden llevar el sistema eléctrico de potencia a un colapso de voltaje, lo que ha ocasionado en algunas oportunidades apagones masivos (llic M., 2007). En los países en desarrollo y en muchos industrializados el control de voltaje se realiza en forma manual mediante la coordinación conjunta entre el centro de despacho y el operador del sistema de transporte, esto puede ocasionar una descoordinación o una equivocada operación causando el colapso de voltaje por eventos en cascada (Ilic M., 2007).

El control automático de voltaje es una técnica ampliamente probada en países como Francia, Italia y en general utilizada total o parcialmente en la Comunidad Económica Europea (Cañizares et al., 2005). El esquema propuesto por éstos se basa en un control jerárquico desacoplado en el tiempo y en el espacio utilizando controladores proporcionales e integrales con base en el generador sincrónico como elemento fundamental de control (Corsi et alt., 2004). La utilización de otros dispositivos de control como son los transformadores con cambio de tomas bajo carga, reactores de líneas, capacitores de compensación, dispositivos FACTS (Sistema de Transmisión Flexible de Corriente Alterna) y la compleja relación existente entre estos elementos, han hecho que se recurra a técnicas de Inteligencia Artificial con el objeto de dotar a los controladores de valoraciones cualitativas y flexibles (Kim et al., 2011; Saxena et al., 2010; Yesuratnam y Srilatha, 2010; Azmy, 2007; Thukaram y Yesuratnam, 2006, Marques et al., 2005; Lin et al., 2003; Bansilal et al. 1997).

A lo largo del tiempo se han desarrollado sistemas expertos que controlan los dispositivos conmutables, dejando la excitación del generador bajo el control del regulador local (AVR Automatic Voltage Regulator) para el transitorio (Bansilal et al., 1995), en otros casos los objetivos de perfil de voltaje y de pérdidas en el sistema eléctrico de potencia se manejan en forma escalonada utilizando primeramente un sistema experto para corregir los niveles en barra y luego un algoritmo de optimización rápida para minimizar las pérdidas (Azmy, 2007; El-Sayed M., 1998), algunos trabajos presentan compensación reactiva en casi todas las barras por lo que el ajuste local conlleva necesariamente a pérdidas mínimas (Pimpa et al., 2002), existen trabajos en los que solamente se corrige el nivel de voltaje en barra (Khaldi M., 2003; Thukaram y Yesuratnam, 2006), otros trabajos no muestran la capacidad de tratar en tiempo real con grandes sistemas (Yesuratnam y Srilatha, 2010), la gran mayoría de ellos operan recalculando la matriz de sensibilidad cuando detectan la anomalía (Kim et al., 2011).

El objetivo del presente trabajo es el desarrollo de un controlador experto que retenga el concepto de nodo piloto y que maneje en forma conjunta el objetivo del perfil de tensiones y la minimización de pérdidas incluyendo el rechazo de carga si es necesario. Se utiliza CLIPS como herramienta fundamental para implementar el experto, se verá a lo largo del trabajo la importancia de utilizar un núcleo específico; como apoyo para el cálculo se utiliza el programa MATLAB, se presentan las diferentes formas de interconexión entre ambos paquetes. El algoritmo desarrollado se simula utilizando dos sistemas de prueba típicos, y los resultados se comparan con otras tecnologías existentes.

El trabajo esta organizado de la siguiente manera: primero se presentan los fundamentos básicos del control de voltaje describiendo los niveles jerárquicos del mismo, la selección del nodo piloto y la descripción detallada de las ecuaciones relacionadas con el mencionado control. Segundo se describen en forma resumida los conceptos más importantes de un sistema experto incluyendo las reglas y bases de conocimiento. Luego se presentan las simulaciones realizadas y se discuten los resultados obtenidos, finalmente se presentan las conclusiones más importantes del trabajo. 


\section{FUNDAMENTOS DEL CONTROL DE VOLTAJE}

\section{Estructura jerárquica del control de voltaje}

El control automático de voltaje, se estructura en tres niveles jerárquicos (Corsi et al., 2004):

Nivel Terciario de aplicación nacional, NVR-TVC (National Voltage Regulation, Tertiary Voltage Control), tiempo de respuesta 5 minutos.

Nivel Secundario de aplicación regional, RVR-SVC (Regional Voltage Regulator, Secondary Voltage Control), tiempo de respuesta 50 segundos.

Nivel Primario de aplicación local, AVR (Automatic Voltage Regulator), tiempo de respuesta instantáneo a 0.5 segundos.

La Fig. 1 ilustra en forma esquemática un diagrama de la estructura jerárquica de control automático de voltaje. Estos niveles no solo están distribuidos espacialmente sino que también lo están temporalmente, el Nivel Primario es de actuación inmediata, mientras que el Nivel Secundario actúa una vez superado el transitorio siendo su constante de tiempo del orden del minuto, y finalmente el Nivel Terciario es el último en actuar y su constante de tiempo se encuentra en el orden de los minutos. Tanto el esquema temporal como el espacial tratan de desacoplar los niveles para evitar oscilaciones.

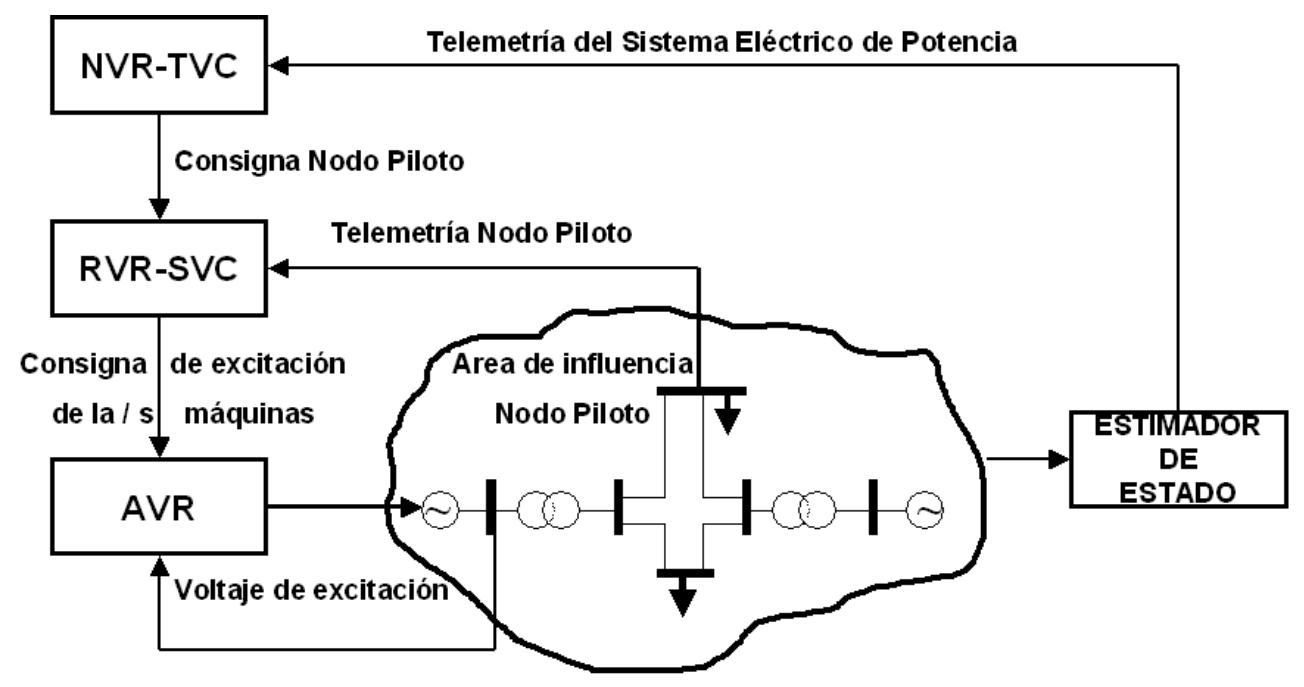

Fig. 1. Diagrama jerárquico del Control de Voltaje.

Selección de los nodos pilotos

La base del sistema está constituida por la elección de nodos pilotos "eléctricamente dominantes" en sus áreas, esto posibilita el manejo acotado de sistemas altamente complejos en cantidad y acoplamiento de variables disminuyendo el orden de las mediciones y de las señales de mando, la idea de un control automático de miles de barras y de líneas de transmisión es muy complejo, muy crítico y puede ser no realista (Corsi et al., 2004; Danelli et al., 2004; Gubina and Curk, 1997).

Para seleccionar los nodos pilotos y las distintas áreas se utilizan los siguientes criterios:

Los nodos deben ser los más "fuertes eléctricamente" en su tipo, deben presentar la mayor capacidad de cortocircuito. 
Deben imponer la variación de voltaje a los nodos eléctricamente vecinos, la inyección de potencia reactiva en ellos debe traducirse en una variación de voltaje también en los nodos eléctricamente próximos.

El acoplamiento entre los distintos nodos pilotos debe ser mínimo.

Con estas consideraciones es posible prevenir el intercambio de potencia reactiva entre áreas vecinas debido a la acción reguladora del sistema.

La elección de las plantas generadoras para el control de voltaje deben ser aquellas próximas a los nodos pilotos con la mayor capacidad de potencia reactiva posible, la simple elección de estas plantas ya plantea una subdivisión de áreas (Danelli et al., 2004; Gubina and Curk, 1997).

El estudio de los nodos pilotos se realiza a partir de las expresiones para el flujo de potencia, la Ec. 1 muestra la expresión matricial lineal del incremento del flujo de potencia activa y reactiva en un sistema de potencia en función del ángulo de voltaje en barra y del módulo de la misma (Kundur, 1994).

$$
\left[\begin{array}{c}
\Delta \mathbf{P} \\
\Delta \mathbf{Q}
\end{array}\right]=\left[\begin{array}{ll}
\frac{\partial \mathbf{P}}{\partial \boldsymbol{\theta}} & \frac{\partial \mathbf{P}}{\partial \mathbf{V}} \\
\frac{\partial \mathbf{Q}}{\partial \boldsymbol{\theta}} & \frac{\partial \mathbf{Q}}{\partial \mathbf{V}}
\end{array}\right]\left[\begin{array}{c}
\Delta \boldsymbol{\theta} \\
\Delta \mathbf{V}
\end{array}\right]
$$

Donde:

$\Delta \mathrm{P}$ Incremento de la potencia activa en la barra.

$\Delta \mathrm{Q}$ Incremento de la potencia reactiva en la barra.

$\Delta \theta$ Incremento del ángulo de voltaje en la barra.

$\Delta \mathrm{V}$ Incremento de voltaje en barra.

La potencia activa y la reactiva inyectada por un nodo $k$-ésimo en una red de $n$ nodos se describen por las Ecs. 2 y 3 las cuales contienen los parámetros del sistema.

$P_{k}=\left|V_{k}\right| \sum_{m=1}^{n}\left(G_{k m}\left|V_{m}\right| \cos \theta_{k m}+B_{k m}\left|V_{m}\right| \operatorname{sen} \theta_{k m}\right)$
$Q_{k}=\left|V_{k}\right| \sum_{m=1}^{n}\left(G_{k m}\left|V_{m}\right| \operatorname{sen} \theta_{k m}-B_{k m}\left|V_{m}\right| \cos \theta_{k m}\right)$

Donde:

$\mathrm{P}_{\mathrm{k}} \quad$ Potencia Activa inyectada en el nodo $k$-ésimo.

$\mathrm{Q}_{k} \quad$ Potencia Reactiva inyectada en el nodo k-ésimo.

$\left|V_{k}\right| \quad$ Módulo de voltaje en el nodo $k$-ésimo.

$\left|\mathrm{V}_{\mathrm{m}}\right| \quad$ Módulo de voltaje en el nodo $m$-ésimo.

$\mathrm{G}_{\mathrm{km}} \quad$ Conductancia entre los nodos k-ésimo y m-ésimo.

$\mathrm{B}_{\mathrm{km}} \quad$ Susceptancia entre los nodos $k$-ésimo y $m$-ésimo.

$\theta_{\mathrm{km}} \quad$ Angulo de fase entre la corriente y el voltaje entre los nodos $k$-ésimo y $m$-ésimo.

Utilizando la notación J para el Jacobiano, la Ec. 1 puede rescribirse en la siguiente forma:

$$
\left[\begin{array}{c}
\Delta \mathbf{P} \\
\Delta \mathbf{Q}
\end{array}\right]=\left[\begin{array}{ll}
\mathbf{J}_{\mathbf{P} \theta} & \mathbf{J}_{\mathbf{P V}} \\
\mathbf{J}_{\mathbf{Q} \theta} & \mathbf{J}_{\mathbf{Q V}}
\end{array}\right]\left[\begin{array}{c}
\Delta \theta \\
\Delta \mathbf{V}
\end{array}\right]
$$

Si a la Ec. 4 se le aplican las simplificaciones para el flujo desacoplado nos queda la Ec. (5). 


$$
\Delta \mathbf{Q}=\mathbf{J}_{\mathrm{QV}} \Delta \mathbf{V}
$$

donde $\mathbf{J}_{\mathrm{Qv}}$ es la submatriz que refleja la dependencia de la potencia reactiva con respecto al voltaje en barra, la cual puede rescribirse como:

$$
\Delta \mathbf{V}=\mathbf{J}_{\mathrm{QV}}^{-1} \Delta \mathbf{Q}
$$

La Ec. 6 es la base para los estudios V-Q los cuales permiten analizar el incremento de voltaje en un nodo debido a la inyección de potencia reactiva en otro, en el presente trabajo los generadores de área inyectan potencia reactiva mediante el control de excitación AVR. Este estudio permite localizar los nodos pilotos, definir las área de influencia de los mismos y seleccionar los controladores.

\section{DESCRIPCION DEL SISTEMA EXPERTO}

\section{Bloques de un sistema experto}

En la Fig. 2 se presentan los bloques que conforman un sistema experto (Giarratano and Riley, 2004), la función que cumple cada uno es la siguiente:

Base de conocimientos: contiene las reglas que definen el comportamiento del experto, las mismas se obtienen de las normas de operación, de los conocimientos que poseen los operadores y de la teoría que gobierna al proceso bajo control.

Base de hechos: es una memoria temporal que refleja el estado de las variables bajo control y de los componentes del sistema de potencia.

Motor de inferencia: es el encargado de comparar los hechos con el antecedente de las reglas, es del tipo IF antecedente THEN consecuente, si el hecho satisface el antecedente se activa la regla y la coloca en la Agenda de ejecución.

Motor de ejecución: Ejecuta la regla ubicada en la prioridad más alta de la agenda, puede modificar el antecedente de una regla no activa o generar una salida para accionar una variable de control. Una tarea importante que tiene este bloque es la resolución de los conflictos de ejecución, puesto que varias reglas pueden estar activas simultáneamente por distintos hechos debe decidir cual se ejecuta, por defecto se suele utilizar el esquema de la primera que entra es la primera que sale (FIFO, First Input - First Output).

SISTEMA EXPERTO

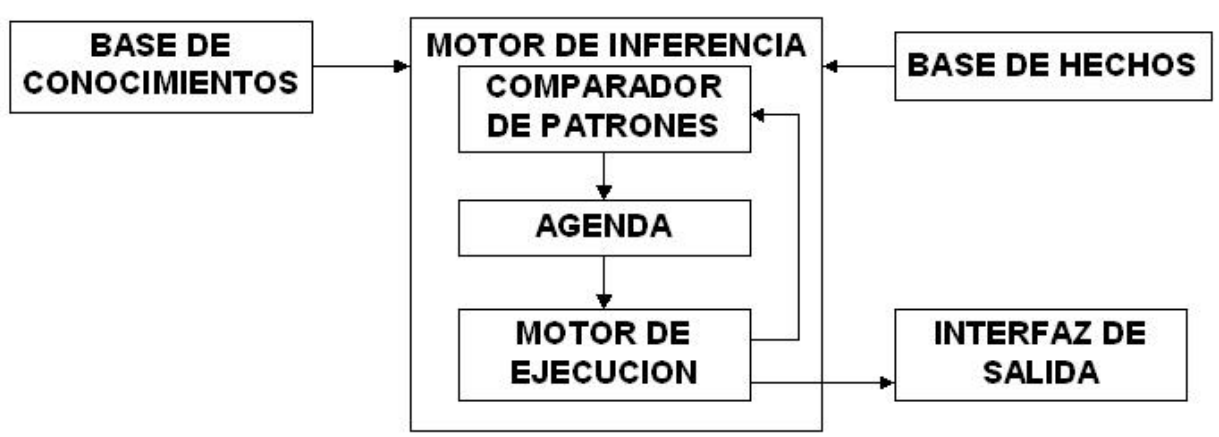

Fig. 2. Bloques de un sistema experto.

Existe un parámetro en la regla denominado prominencia (salience), el cual le permite al programador controlar el orden en que se ejecuta dicha regla, la utilización repetitiva del mismo 
puede significar una base de conocimientos mal definida o indicar que quizás sea mejor recurrir a un lenguaje de procedimientos para programar el algoritmo de control (Giarratano and Riley, 2004).

\section{Lenguajes basados en reglas: CLIPS}

Definidas las reglas se podría recurrir a un lenguaje de procedimientos con el objeto de programar el algoritmo de control, esto trae aparejado un primer y gran problema, si se agrega una regla nueva se deberá modificar el código del algoritmo a los fines de dar cabida a este nuevo conocimiento, además la ineficiencia en la escritura del código de la estructura IF ... THEN ... es alta. En lugar de ello se emplea un lenguaje basado en reglas como CLIPS (C Language Integrated Production System - Sistema de Producción Integrado en Lenguaje C), desarrollado por la NASA (National Aeronautics and Space Administration) el cual es de uso gratuito y mantenido por su autor original Gary Riley. CLIPS soporta los paradigmas de programación estructurada, por objetos y de sistemas expertos (CLIPS, 2012).

Utilizar un lenguaje de esta naturaleza obliga a definir la base de conocimientos en forma precisa y que sean los conocimientos del sistema expresados en reglas, los que guíen la lógica de la ejecución, si se agrega una nueva regla se deberá revisar su coherencia con las demás pero no será necesario reprogramar el núcleo.

La expresión lógica de las reglas en CLIPS es la siguiente:

IF ciertas condiciones son verdaderas THEN ejecute las siguientes acciones.

La sintaxis de las reglas en CLIPS es la mostrada en la Fig. 3.

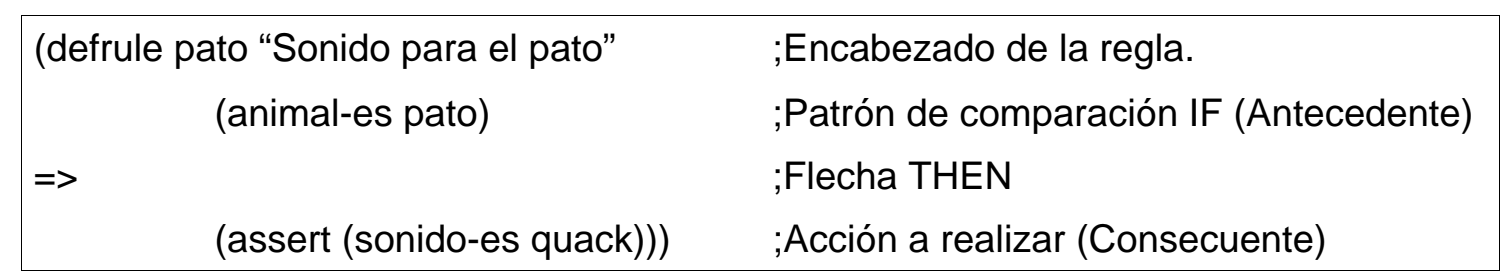

Fig. 3. Sintaxis para una regla en CLIPS.

Para mayor claridad la reglas se escribirán de la siguiente forma:

R1: Si el animal es pato entonces el sonido es "quack".

Bases de conocimientos y reglas aplicadas al control de voltaje

Las reglas son la expresión formal de la base de conocimientos que definen al algoritmo de control, es necesario a partir de este conocimiento construir el conjunto de reglas que serán gestionadas por el sistema experto, es posible obtener este conocimiento de los operadores y/o de las normas de operación las cuales fijan procedimientos ante contingencias, esta base de conocimientos se puede ampliar y validar con la teoría sobre la que se cimienta el proceso bajo control (Kundur, 1994). Para el presente trabajo se ha tomado como referencia las normas de operación de AESO para el control de voltaje (AESO, 2010), de esta norma y de los fundamentos teóricos surge la base de conocimientos contenida en la Tabla 1.

La base de conocimientos se forma en algunos casos con los datos del fabricante como en C2, con estudios teóricos como en $\mathrm{C} 1$ y con conocimientos heurísticos como en C10. A partir de la base de conocimientos podemos definir el conjunto de reglas de la Tabla 2. 
Tabla 1: Base de conocimientos.

\begin{tabular}{|l|l|}
\hline C1 & $\begin{array}{l}\text { Nodos pilotos y lista de controladores asociados definidos de acuerdo al estudio } \\
\text { V-Q. }\end{array}$ \\
\hline C2 & $\begin{array}{l}\text { Límites máximos, mínimos, incremento y cantidad de conmutaciones permitidas por los } \\
\text { controladores. }\end{array}$ \\
\hline C3 & Lista de preferencia de los controladores. \\
\hline C4 & Niveles de voltaje permitidos en las barras del sistema. \\
\hline C5 & $\begin{array}{l}\text { Si el voltaje en un barra sube por encima (baja por debajo) del límite permitido los } \\
\text { dispositivos de control deben ser conmutados o ajustados para recuperar el perfil de } \\
\text { tensiones. }\end{array}$ \\
\hline c6 & $\begin{array}{l}\text { Es más eficiente aplicar la compensación reactiva en forma local. Si la capacidad del } \\
\text { compensador local se agota se debe seleccionar el más sensible que le sigue en la lista. }\end{array}$ \\
\hline c7 & $\begin{array}{l}\text { Si el voltaje en barra es bajo (alto) la toma del transformador local debe ser aumentada } \\
\text { (disminuida) para corregir el problema; sin embargo se debe tener en cuenta que esto } \\
\text { puede causar el efecto inverso en otras barras del sistema. }\end{array}$ \\
\hline c8 & $\begin{array}{l}\text { La excitación del generador debe aumentar (disminuir) para resolver una baja (suba) de } \\
\text { voltaje en barra. }\end{array}$ \\
\hline c9 & $\begin{array}{l}\text { Para minimizar las pérdidas y estar mejor preparados ante una contingencia es preferible } \\
\text { que las tensiones en barra estén próximas a su límite superior. }\end{array}$ \\
\hline C10 & $\begin{array}{l}\text { Es deseable balancear la potencia reactiva entre los generadores localizados } \\
\text { eléctricamente próximos; para lograr este objetivo el voltaje en terminales de los } \\
\text { generadores debe ser operado simultáneamente de tal forma que la variación en un } \\
\text { generador no sea absorbida por otro. }\end{array}$ \\
\hline C11 & $\begin{array}{l}\text { Cuando el recurso de potencia reactiva resulte insuficiente se deberá recurrir al rechazo } \\
\text { de carga para resolver el inconveniente. }\end{array}$ \\
\hline
\end{tabular}

Tabla 2: Reglas basadas en la tabla de conocimientos.

\begin{tabular}{|l|l|}
\hline R1 & $\begin{array}{l}\text { IF el voltaje en barra es violado THEN formar la lista de violaciones } A N D \text { inicializar el } \\
\text { incremento de los controladores a cero. }\end{array}$ \\
\hline R2 & IF no existe una violación THEN ejecutar el próximo ciclo. \\
\hline R3 & $\begin{array}{l}\text { IF la lista de violaciones no está vacía THEN seleccionar de la lista la peor violación AND } \\
\text { tomar de la lista de controladores asociados a este nodo el primer controlador } A N D \\
\text { aumentar o disminuir según corresponda la consigna del controlador } A N D \text { guardar el } \\
\text { valor en la tabla de estado de los controladores. }\end{array}$ \\
\hline R4 & $\begin{array}{l}\text { IF el incremento o decremento de un controlador causa que el voltaje de algún nodo bajo } \\
\text { control esté fuera del rango de operación THEN disminuir o aumentar la consigna del } \\
\text { controlador correspondiente AND seleccionar el próximo controlador de la lista de } \\
\text { controladores AND actualizar la tabla de estado de los controladores. }\end{array}$ \\
\hline R5 & $\begin{array}{l}\text { IF el incremento o decremento de un controlador alcanza el límite del controlador OR } \\
\text { viola los valores de conmutación THEN bloquear el controlador. }\end{array}$ \\
\hline R6 & $\begin{array}{l}\text { IF el nodo bajo análisis corrige su valor } A N D \text { si no existe violación en otro nodo como } \\
\text { consecuencia del incremento o decremento del controlador THEN fijar como nuevo punto } \\
\text { de operación del controlador el valor calculado } A N D \text { actualizar la lista de violaciones. }\end{array}$ \\
\hline R7 & $\begin{array}{l}\text { IF todos los controladores del área han sido ajustados } A N D \text { no existen nodos en la lista } \\
\text { de violaciones THEN ejecutar el próximo ciclo. }\end{array}$ \\
\hline R8 & $\begin{array}{l}\text { IF ocurre un incremento sucesivo de todos los controladores } A N D \text { algún nodo excede el } \\
\text { límite máximo permitido THEN concluir el ciclo. }\end{array}$ \\
\hline R9 & $\begin{array}{l}\text { IF se ha disminuido la consigna de un controlador } A N D \text { todos los nodos están en su nivel } \\
\text { correcto THEN concluir el ciclo. }\end{array}$ \\
\hline R10 & $\begin{array}{l}\text { IF todos los controladores del nodo han sido utilizados } A N D \text { el nivel del nodo sigue bajo } \\
\text { THEN rechazar carga del nodo en el porcentaje programado. }\end{array}$ \\
\hline R11 & $\begin{array}{l}\text { IF el nivel del nodo está por encima del rango permitido } A N D \text { el nodo presenta rechazo } \\
\text { de carga THEN reconectar carga del nodo en el porcentaje programado. }\end{array}$ \\
\hline
\end{tabular}


El número de la regla no implica necesariamente el orden de su ejecución, la regla será ejecutada según el hecho que satisface el antecedente, la prominencia de la misma o el esquema de resolución de conflictos que se haya elegido.

Una característica importante del controlador es que se basa en estructuras de datos para la gestión de control, esto hace que si el sistema crece solo deban actualizarse estas bases sin necesidad de cambiar la lógica del sistema experto y sus reglas, esto último podría ser necesario si el cambio es muy grande e involucra algún nuevo concepto que modifique la base de conocimientos. En las Tablas 3 y 4 , se detallan la estructura de dos bases, la relativa a los controladores, y a los nodos pilotos respectivamente.

\section{Controlador experto}

En la Fig. 4 se presenta el sistema de control experto propuesto, la telemetría de los nodos pilotos se ha puesto en la figura fuera del estimador de estado a los fines de resaltar su utilización. En esta figura también se aprecia el motor de calculo que contiene las subrutinas que realizan los cómputos eléctricos tales como el flujo de potencia y las pérdidas del sistema. La implementación se realizó en MATLAB.

Tabla 3: Matriz de datos de los controladores.

\begin{tabular}{|c|c|}
\hline Registro & LC[c1 m1 M1 r1 d1 e1, c2 m2 M2 r2 d2 e2, .., cn mn Mn rn dn en] \\
\hline \multirow{6}{*}{ Parámetros } & cn = Controlador número. \\
\hline & mn = Valor mínimo del controlador. \\
\hline & Mn = Valor máximo del controlador. \\
\hline & rn = Referencia actual del controlador. \\
\hline & dn $=$ Delta del controlador \\
\hline & en = Estado del controlador. \\
\hline
\end{tabular}

Tabla 4: Matriz de datos de los nodos pilotos

\begin{tabular}{|c|l|}
\hline Registro & LNP $[\mathrm{n} 1 \mathrm{c} 1 \mathrm{c} 2 \ldots \mathrm{cn}, \mathrm{n} 2 \mathrm{c1} \mathrm{c} 2 \ldots \mathrm{cn}, \mathrm{nn} \mathrm{c} 1 \mathrm{c} 2 \ldots \mathrm{cn}]$ \\
\hline \multirow{2}{*}{ Parámetros } & $\mathrm{nn}=$ Número nodo. \\
\cline { 2 - 2 } & $\mathrm{cn}=$ Controlador nodo. \\
\hline
\end{tabular}

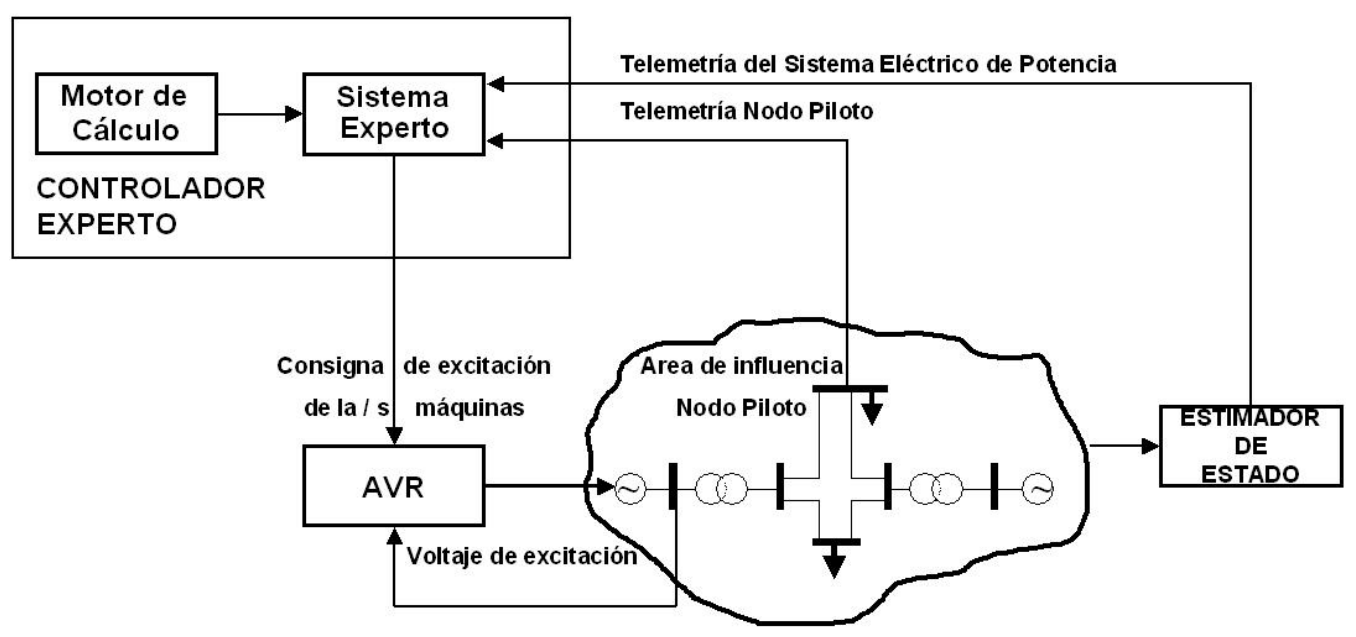

Fig. 4. Sistema de control experto basado en nodos pilotos. 
En la Tabla 5 se presentan las distintas formas de vincular MATLAB con CLIPS, para el presente trabajo se ha elegido la opción 3, las rutinas de cálculo se escriben en MATLAB como funciones externas a CLIPS, reteniendo la interfaz gráfica de CLIPS para el desarrollo del sistema experto (MATLAB, 2012).

Mayor detalles de estos mecanismos pueden obtenerse del Manual de Referencia de CLIPS Volumen II (Advanced Programming Guide) y en la ayuda de MATLAB (External Interfaces Calling MATLAB from $C$ and Fortran Programs).

A continuación se presenta un diagrama de flujo que resume la lógica de funcionamiento del controlador, Fig. 5, se debe tener en cuenta que esta lógica está embebida en las reglas.

Tabla 5: Métodos de vinculación CLIPS - MATLAB

\begin{tabular}{|l|l|l|}
\hline 1 & Archivos. & $\begin{array}{l}\text { Se trata de una primitiva forma de comunicación entre } \\
\text { programas, utiliza archivos de texto para compartir } \\
\text { información, CLIPS no permite el intercambio XML. }\end{array}$ \\
\hline 2 & $\begin{array}{l}\text { Objetos de comunicación } \\
\text { Cliente - Servidor. }\end{array}$ & $\begin{array}{l}\text { Permite la utilización de protocolos IP, DDE, OPC o } \\
\text { propietarios. }\end{array}$ \\
\hline 3 & $\begin{array}{l}\text { Funciones externas de } \\
\text { CLIPS. }\end{array}$ & $\begin{array}{l}\text { Se trata de una función externa a CLIPS escrita en "C" } \\
\text { que luego se compila y vincula con éste. }\end{array}$ \\
\hline 4 & CLIPS embebido. & $\begin{array}{l}\text { Utiliza el ejecutable de CLIPS como una rutina "C" } \\
\text { disponiendo de todas sus primitivas. }\end{array}$ \\
\hline
\end{tabular}

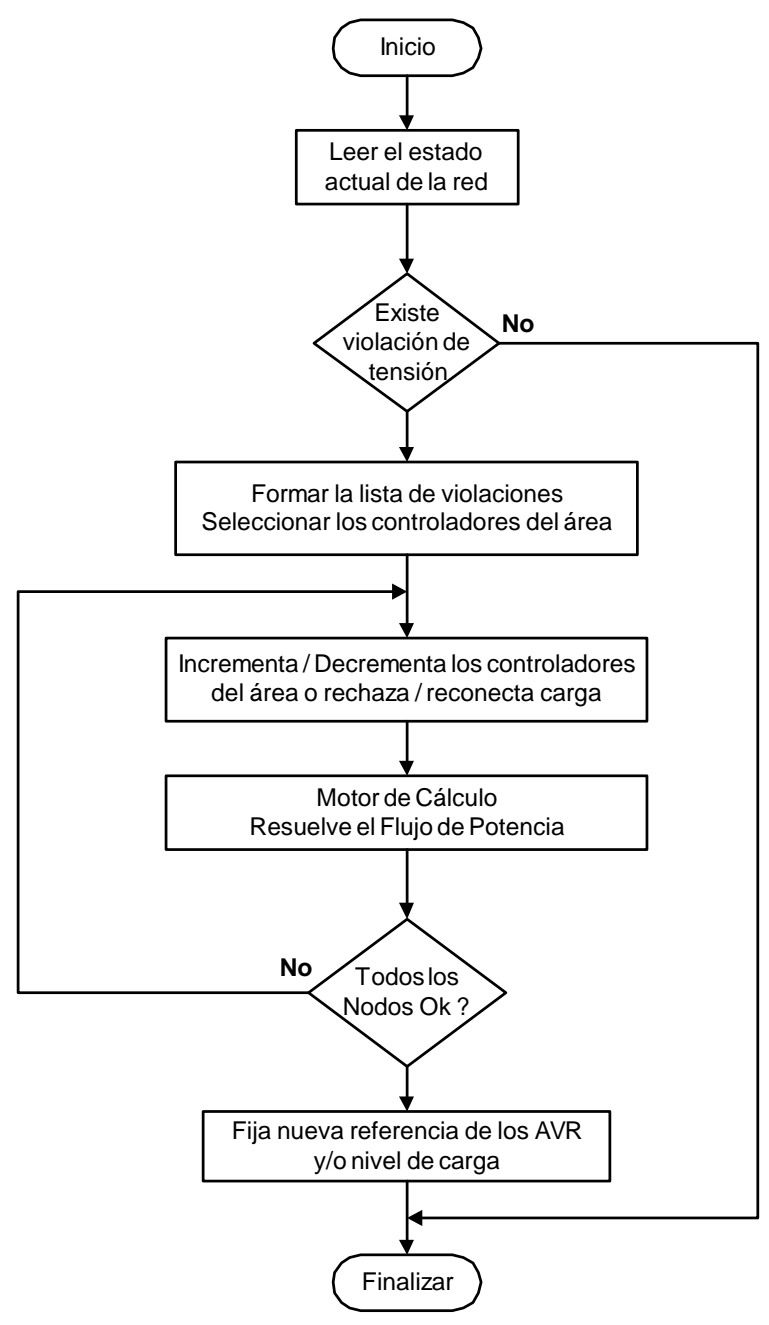

Fig. 5. Diagrama lógico del controlador experto. 


\section{RESULTADOS DE LA SIMULACION}

Para verificar el comportamiento del controlador experto se simuló el control del sistema de tres generadores y nueve barras del Western Electricity Coordinating Council (Anderson and Fouad, 2003). La Fig. 6 presenta un diagrama unifilar del sistema de prueba, el mismo representa una región a controlar, siendo la barra 9 el nodo piloto y el voltaje en terminales de los tres generadores las variables de control. Un sistema más grande se estructura en áreas similares.

En la Tabla 6 se presentan los resultados obtenidos para este sistema simulando tres contingencias diferentes.

Caso I: Representa el sistema con una demanda del $20 \%$, y se produce un incremento de demanda al $150 \%$.

Caso II: Representa el sistema con una demanda del $150 \%$ y se produce un caída de la misma, llevando ésta al $20 \%$.

Caso III: presenta el sistema con la demanda pico y se produce la desconexión de la línea 4 - 9.

Si bien estas contingencias implican transitorios con una alta posibilidad de inestabilidad, se supone que éstos han sido sorteados con éxito por la combinación AVR - PSS (Automatic Voltage Regulator - Power System Stabilizer) y que se está en la etapa de estabilidad de voltaje en el largo plazo ante una gran perturbación (Kundur et al., 2004).

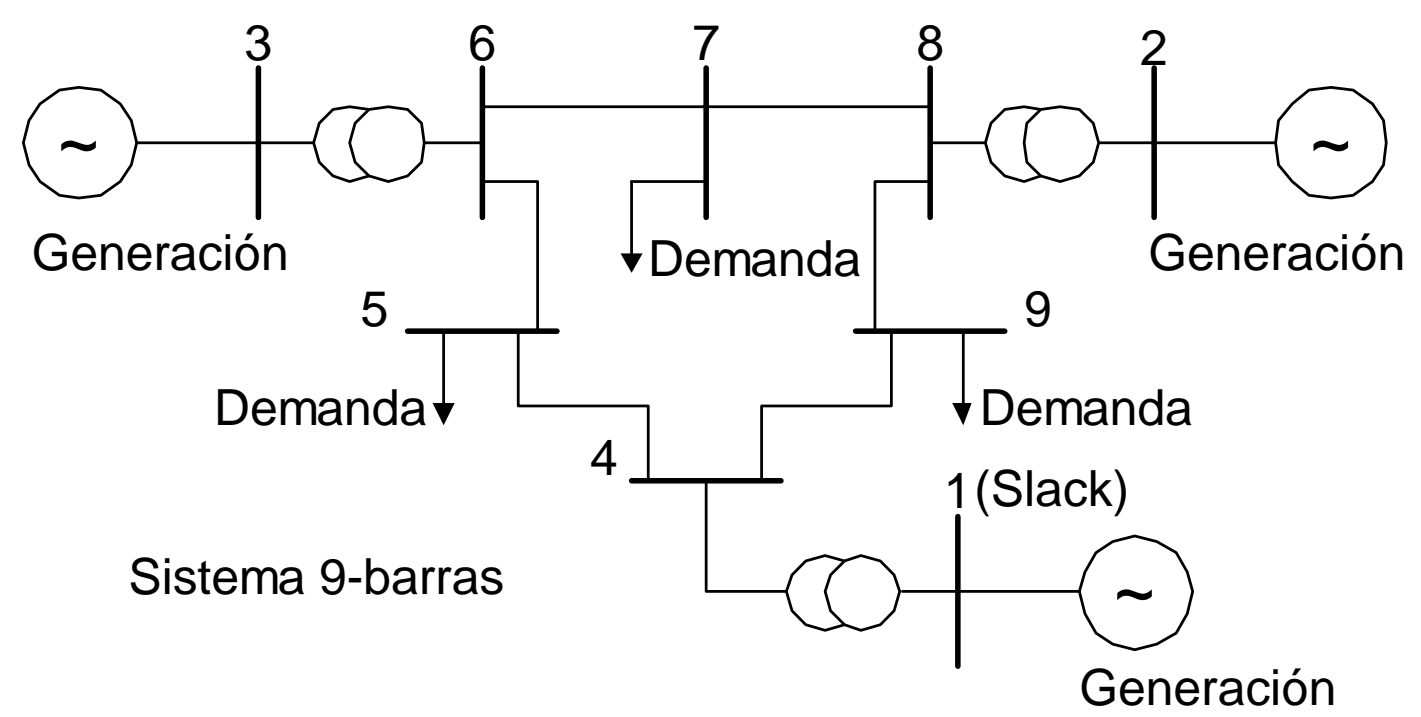

Fig. 6. Sistema WECC de 9 barras y 3 generadores.

La respuesta del controlador experto se compara con la solución brindada por un flujo óptimo de potencia (OPF) y la dada por un algoritmo genético (GA). El algoritmo genético programado tiene una estructura clásica, es del tipo continuo y no se ha recurrido a técnicas avanzadas para acelerar su procesamiento (Haupt and Haupt, 2004). 
Tabla 6: Resultados de la simulación.

\begin{tabular}{|c|c|c|c|c|c|c|c|c|c|c|c|c|}
\hline & \multicolumn{4}{|c|}{ Caso I } & \multicolumn{4}{|c|}{ Caso II } & \multicolumn{4}{|c|}{ Caso III } \\
\hline & Inicial & OPF & Experto & GA & Inicial & OPF & Experto & GA & Inicial & OPF & Experto & GA \\
\hline & \multicolumn{12}{|c|}{ Variables de control } \\
\hline \% Carga & $20->150$ & 150 & 150 & 150 & $150->20$ & 20 & 20 & 20 & 100 & 100 & 100 & 100 \\
\hline V1 [pu] & 1.011 & 1.050 & 1.041 & 1.046 & 1.050 & 1.011 & 1.010 & 1.008 & 1.050 & $\mathrm{NC}$ & 1.050 & 1.046 \\
\hline V2 [pu] & 1.025 & 1.050 & 1.045 & 1.045 & 1.050 & 1.025 & 1.020 & 1.020 & 1.050 & $\mathrm{NC}$ & 1.050 & 1.049 \\
\hline \multirow[t]{2}{*}{ V3 [pu] } & 1.021 & 1.050 & 1.041 & 1.050 & 1.050 & 1.021 & 1.020 & 1.017 & 1.040 & $\mathrm{NC}$ & 1.040 & 1.048 \\
\hline & \multicolumn{12}{|c|}{ Variables controladas } \\
\hline V5 [pu] & 0.952 & 0.993 & 0.983 & 0.990 & 1.085 & 1.050 & 1.048 & 1.046 & 1.034 & $\mathrm{NC}$ & 1.036 & 1.037 \\
\hline V7 [pu] & 0.978 & 1.012 & 1.003 & 1.008 & 1.079 & 1.050 & 1.047 & 1.045 & 1.011 & NC & 1.027 & 1.031 \\
\hline V9 [pu] & 0.927 & 0.968 & 0.958 & 0.964 & 1.081 & 1.046 & 1.043 & 1.042 & 0.873 & NC & 0.951 & 0.960 \\
\hline & \multicolumn{12}{|c|}{ Indices de eficiencia } \\
\hline $\begin{array}{l}\text { Ploss } \\
\text { [MW] }\end{array}$ & & 8.703 & 8.904 & 8.780 & & 0.279 & 0.280 & 0.279 & & NC & 4.781 & 4.446 \\
\hline te $[\mathrm{s}]$ & & 0.23 & 0.13 & 8.89 & & 0.27 & 0.10 & 9.53 & & $\mathrm{NC}$ & 0.13 & 11.4 \\
\hline it & & 12 & 3 & 67 & & 13 & 3 & 70 & & $\mathrm{NC}$ & 7 & 85 \\
\hline $\mathrm{rc}$ & & $0 \%$ & $0 \%$ & $0 \%$ & & $0 \%$ & $0 \%$ & $0 \%$ & & $\mathrm{NC}$ & $25 \%$ & $28 \%$ \\
\hline
\end{tabular}

La función de ajuste desarrollada para el algoritmo genético está dada por la Ec. 7 .

$$
F=P_{\text {loss }}+K_{v} \sum_{i \in N} h\left(v_{i}\right)+K_{L} \sum_{i \in N_{P Q}} g\left(L_{i}\right)
$$

Donde

$\begin{array}{ll}\mathrm{F} & \text { Función de ajuste a minimizar (Fitness function). } \\ \mathrm{P}_{\text {loss }} & \text { Pérdidas activas en el sistema. } \\ \mathrm{K}_{\mathrm{V}} & \text { Coeficiente de penalización para las tensiones de barra. } \\ \mathrm{K}_{\mathrm{L}} & \text { Coeficiente de penalización para el rechazo de carga. } \\ \mathrm{h}\left(\mathrm{v}_{\mathrm{i}}\right) & \text { Función de penalización para las tensiones de barra. } \\ \mathrm{g}\left(\mathrm{L}_{\mathrm{i}}\right) & \text { Función de penalización para el rechazo de carga. } \\ \mathrm{N} & \text { Conjunto de todas las barras del sistema. } \\ \mathrm{N}_{\mathrm{PQ}} & \text { Conjunto de todas las barras de carga del sistema. }\end{array}$

Siendo las funciones de penalización las dadas por las Ecs. 8 y 9.

$$
\begin{aligned}
& h\left(v_{i}\right)=\left\{\begin{array}{lll}
\left(v_{i}-v_{i}^{\text {máx }}\right)^{2} & \text { si } & v_{i}>v_{i}^{\text {máx }} \\
\left(v_{i}^{\text {mín }}-v_{i}\right)^{2} & \text { si } & v_{i}<v_{i}^{\text {mín }} \\
0 & \text { si } & v_{i}^{\text {mín }} \leq v_{i} \leq v_{i}^{\text {máx }}
\end{array}\right. \\
& g\left(L_{i}\right)=\left\{\begin{array}{lll}
\left(1-L_{i}\right)^{2} & \text { si } & L_{i}<1 \\
0 & \text { si } & L_{i}=1
\end{array}\right.
\end{aligned}
$$

$\mathrm{L}_{\mathrm{i}}=$ Carga Normalizada del nodo $\mathrm{i}$.

El algoritmo genético debe resolver el problema de minimizar $F$ sujeta a las restricciones planteadas en las Ecs. 8 y 9.

El cálculo del flujo óptimo de potencia se basa en el paquete MatPower, provisto en forma gratuita para investigadores y educadores por el Power Systems Engineering Research Center, se puede utilizar desde MATLAB en modo comando o en modo función (Zimmerman et al., 2012).

Por último, de forma de valorar las prestaciones en tiempo real se realiza la simulación de un cuarto caso, cuyos resultados se presentan en la Tabla 7. El sistema de prueba utilizado para estas simulación es de 30 barras y seis generadores denominado IEEE30 (Alsac and Stott, 1974; 
Zimmerman et al., 2012). Se omite el diagrama unifilar con el objeto de ahorrar espacio y los valores de la tabla se limitan a las consignas de generación, a la comparación entre sistema experto y flujo óptimo de potencia y a los nodos con problemas después de la contingencia, línea $4-6$ fuera de servicio.

\section{ANÁLISIS DE LOS RESULTADOS}

Para analizar los datos presentados en la Tabla 6 se debe tener en cuenta que el Caso II es similar al Caso I, la diferencia radica en que el Caso II implica una disminución de la tensiones de control mientras que en el Caso I se debe realizar un incremento de éstas, esto permite demostrar que el algoritmo trabaja en los dos sentidos, fuera de esto las conclusiones son similares.

Caso I: El sistema tiene una demanda del 20\%, por lo tanto las tensiones de generación están en un nivel bajo, como consecuencia del incremento súbito del consumo el nodo V9 experimenta un descenso por debajo del nivel permitido que es de 0.95 pu (Por unidad). Todos los algoritmos sugieren un incremento de las tensiones de control logrando recomponer el nivel en el nodo en un valor mayor a los $0.95 \mathrm{pu}$.

Se han fijado cuatro índices que permiten medir la efectividad de los controles, las pérdidas activas del sistema (Ploss), el tiempo de ejecución (te), la cantidad de iteraciones (it) y el rechazo de carga ( $\mathrm{rc}$ ).

Tabla 7: Sistema de 30 barras y 6 generadores.

\begin{tabular}{|c|c|c|c|}
\hline & \multicolumn{3}{|c|}{ Caso IV } \\
\hline & Inicial & OPF & Experto \\
\hline & \multicolumn{3}{|c|}{ Variables de control } \\
\hline V1[pu] & 0.974 & 0.990 & 1.044 \\
\hline V2[pu] & 0.970 & 0.985 & 1.050 \\
\hline V13[pu] & 1.050 & 1.050 & 1.050 \\
\hline V22[pu] & 0.996 & 0.998 & 1.046 \\
\hline V23[pu] & 1.011 & 1.016 & 1.041 \\
\hline \multirow[t]{2}{*}{ V27[pu] } & 1.050 & 1.050 & 1.050 \\
\hline & \multicolumn{3}{|c|}{ Variables controladas } \\
\hline V7[pu] & 0.944 & 0.955 & 1.010 \\
\hline \multirow[t]{2}{*}{ V8[pu] } & 0.941 & 0.950 & 1.002 \\
\hline & \multicolumn{3}{|c|}{ Indices de eficiencia } \\
\hline Ploss[MW] & & 2.698 & 2.593 \\
\hline te[s] & & 0.7 & 0.54 \\
\hline it & & 20 & 8 \\
\hline rc & & $0 \%$ & $0 \%$ \\
\hline
\end{tabular}

Puede apreciarse que el nivel de pérdidas es similar para los tres controles, siendo en el caso del sistema experto superior en $2.3 \%$ con respecto al OPF, esto puede mejorarse si se considera un paso menor en el incremento de la señal de control, permitiría elevar el nivel sin excederlo, pero aumentaría las iteraciones y por ende el tiempo de ejecución del algoritmo.

Con respecto al tiempo de ejecución y habiéndose efectuado la simulación con la misma plataforma, el algoritmo experto presenta el menor de todos y está en el orden del flujo óptimo de potencia, siendo también la cantidad de iteraciones la menor. Se destaca el comportamiento del algoritmo genético que presenta el peor desempeño en relación a estos dos índices, se ha hecho mención ya al hecho de que la programación ha sido estándar, es posible sintonizar varios parámetros, para este estudio se han tomado los valores típicos. 
Caso II: Se pueden obtener conclusiones similares, aquí debido a la baja súbita de la demanda los nodos bajo control experimentan un aumento en el voltaje que excede el límite permitido de 1.05 en pu. En ambos casos no ha sido necesario recurrir al rechazo de carga ( $\mathrm{rc}=0 \%$ ), con la corrección de los valores de voltaje de generación ha sido suficiente para corregir los valores en los nodos controlados.

Caso III: El nodo piloto V9 experimenta una disminución de voltaje a 0.873 pu, el flujo óptimo de potencia utilizado no puede encontrar una solución (NC - No Converge) en un número de iteraciones razonable, mientras que los otros dos algoritmos si lo hacen. Aquí se debe recurrir al rechazo de carga para estabilizar las tensiones. Los niveles de voltaje sugeridos para los generadores de control son similares, también lo son las tensiones corregidas de los nodos bajo control, la mayor diferencia se encuentra en los tiempos de ejecución y en la cantidad de iteraciones, siendo el controlador experto quién presenta los mejores índices. Si bien las pérdidas son mayores en el experto, en el orden del 7\% con respecto al GA, el rechazo de carga es menor, $3 \%$.

Del análisis precedente se desprende que un controlador basado en un sistema experto resulta competitivo en relación a las otras propuestas, más aún cuando se desea incorporar a la ley de control objetivos y restricciones difícilmente expresables en forma analítica, rechazar generación como consecuencia de la falta de capacidad de transporte, establecer un orden de prioridad en los controles a utilizar y/o fijar un límite a las conmutaciones de los controladores.

En relación a otros algoritmos expertos para el control de voltaje, el concepto de nodo piloto permite prescindir del cálculo de la matriz de sensibilidad cada vez que se produce una contingencia. La aplicación de la norma AESO posibilita la gestión simultánea del nivel y de las pérdidas sin necesidad de recurrir a un algoritmo de dos pasos. La utilización de un paquete específico para la programación de sistemas expertos permite que los cambios y/o ampliaciones del sistema se vean reflejados en la base de datos sin necesidad de cambiar la lógica de ejecución, puede ser necesario introducir una nueva regla o verificar las consistencia de las existentes si se varía algún concepto de la base de conocimientos.

Con respecto a la aplicación en tiempo real se deben considerar dos aspectos, el desacople temporal entre AVR y SVC que es del orden de los $50 \mathrm{seg}$. y el tamaño de la región controlada por el nodo piloto. En función de estos dos parámetros se puede establecer la capacidad del controlador para su respuesta en tiempo real.

Caso IV: Ios resultados de esta simulación muestra que se duplican la cantidad de controladores y se triplica la cantidad de los nodos a controlar en relación al sistema de la Fig. 5. manteniéndose los índices de eficiencia dentro de los valores anteriores. La constante de tiempo del lazo de control SVC para desacoplar a éste del nivel primario y del nivel terciario es 100 veces superior al tiempo de ejecución del algoritmo experto (te), siendo posible la operación en tiempo real.

\section{CONCLUSIONES}

Este trabajo propone un método de control secundario de voltaje basado en un sistema experto considerando el concepto de nodo piloto. El algoritmo implementado se simuló utilizando dos sistemas de prueba típicos incluyendo distintos tipos de contingencias. De acuerdo a los resultados obtenidos y al análisis de los mismos se pueden extraer las siguientes ventajas del método desarrollado:

Si se presenta una contingencia no es necesario recalcular la matriz de sensibilidad debido a la utilización del concepto de nodo piloto. Es posible, ajustando coordinadamente todos los controladores del área, corregir las desviaciones de los nodos afectados y minimizar las pérdidas del sistema en forma simultánea sin recurrir a un algoritmo de dos pasos. Ante cambios topológicos del sistema solo se necesitan actualizar las bases de datos sin necesidad de cambiar la lógica de control, puede ser necesario un cambio de reglas si se incluyen nuevos conocimientos. 
El controlador resulta apto para la operación en tiempo real si se compara el tiempo de ejecución del algoritmo con la constante de tiempo del control secundario de voltaje. Emplear un lenguaje basado en reglas como CLIPS obliga al correcto planteo de las reglas ya que si así no fuera se presentarían problemas de ejecución. La utilización de un sistema experto permite incluir en la ley de control valoraciones cualitativas y aún cuantitativas de difícil formulación analítica. Es sencillo incluir otro tipo de controlador, como por ejemplo dispositivos FACTS, ya que con definirlo en la base de datos, asignarlo a un nodo piloto y a una región el mismo queda activo. Parte del control terciario de voltaje como es el despacho óptimo de la potencia reactiva con minimización de pérdidas se calcula en el controlador experto.

\section{REFERENCIAS}

AESO, Operating Policies and Procedures: Routine System Operations OPP 702 Voltage Control, http://www.aeso.ca/rulesprocedures/9073.html, Acceso: 19 Enero (2012).

Alsac O. y Stott B., Optimal Load Flow with Steady State Security, IEEE Transactions on Power Apparatus and Systems: 93(3), 745-751 (1974).

Anderson P. M. y Fouad A. A., Power System Control and Stability, $2^{a}$ edición, 37-39, WileyInterscience, IEEE Press, USA (2003).

Azmy A. M., "Optimal Power Flow to Manage Voltage Profiles in Interconnected Networks Using Expert Systems", IEEE Transactions on Power Systems, 22(4), 1622-1628 (2007).

Bansilal, Thukaram D. y Parthasarathy K., An expert system for alleviation of network overloads, Electric Power System Research: 40(2), 143-153 (1997).

Bansilal, Thukaram D. y Parthasarathy K., An Expert System for Voltage Control in a Power System Network, IEEE Proceedings of Energy Management and Power Delivery: 1, 364-369 (1995), http://ieeexplore.ieee.org, Acceso: 17 Enero (2012).

Cañizares C., Cavallo C., Pozzi M. y Corsi S., Comparing Secondary Voltage Regulation and Shunt Compensation for Improving Voltage Stability and Transfer Capability in the Italian Powwer System, Electric Power Systems Research: 73(1), 67-76 (2005).

CLIPS, A Tool for Bulding Expert Systems: User's Guide., http://clipsrules.sourceforge.net/, Acceso: 18 Enero (2012).

Corsi S., Pozzi M., Sabelli C. y Serrani A., The Coordinated Automatic Voltage Control of the Italian Transmission Grid-Part I: Reasons of the Choice and Overview ot The Consolidated Hierarchical System, IEEE Transaction on Power Systems: 19(4), 1723-1732 (2004).

Danelli A., Denegri G. B., Invernizzi M., Pozzi M. y Serra P., Electric Power System Monitoring: Phenomenon Independent Positioning of a Constrained Number of PMUs, Bulk Power System Dynamics and Control - VI IREP, Cortina D'Ampezzo, Italy (2004), http://areeweb.polito.it/eventi/irep2004/Session\%20A2/A2.3.pdf, Acceso: 17 Enero (2012).

El-Sayed M., Rule-based approach for real-time reactive power control in interconnected power systems, Expert Systems with Applications: 14(3), 355-360 (1998).

Giarrtano J. y Riley D., Expert Systems: Principles and Programming, $4^{\mathrm{a}}$ edición, 1-578, Thomson Course Technology, New York, USA (2004).

Gubina F. y Curk J., Modular secondary voltage control based on local information, European Transactions on Electrical Power: 7(3), 179-184 (1997). 
Haupt R. L. y Haupt S. E., Practical Genetic Algorithms, $2^{a}$ edición, 95 - 148, Wiley-Interscience, John Wiley \& Sons, New Jersey, USA (2004).

Ilic M., From Hierarchical to Open Access Electric Power Systems, Proceedings of the IEEE: 95(5), 1060-1084 (2007).

Khaldi M., An Intelligent Cognitive Expert System for Voltage Control in Power Systems, Proceedings of 2003 IEEE Conference on Control Applications: 1, 319-324 (2003), http://ieeexplore.ieee.org, Acceso: 17 Enero (2012).

Kim K., Yu W., Lee E., Wang I., Kang H., Shin J. y Lee H., "Development of an Intelligent Voltage Control System for Jeju Island in Korea", Journal of International Council on Electrical Engineering, 1(2), 156-162 (2011).

Kundur P., Power System Stability and Control, Edición EPRI (Electric Power Research Institute), 627-631, McGraw-Hill, New York, USA (1994).

Kundur P., Paserba J., Ajjarapu V., Anderson G., Bose A., Cañizares C., Hatziargyriou N., Hill D., Stankovic A., Taylor C., Van Cutsem T., y Vittal V., Definition and Classification of Power System Stability, IEEE Transaction on Power Systems: 19(2), 1387 -1401 (2004).

Lin M., Rayudu R. K. y Samarasinghe S., A Review of Voltage/VAR Control, AUPEC2003 (Australasian Universities Power Engineering Conference) ISBN 0-473-09867-9 paper No 126, http://itee.uq.edu.au/ aupec/aupec03/papers.htm, Acceso: 17 Enero (2012).

Marques A. B., Taranto G. y Falcão D., A Knowledge-Based System for Supervision and Control of Regional Voltage Profile and Security, IEEE Transactions on Power Systems: 20(1), 400-407 (2005).

MATLAB, The Languaje of Technical Computing: User's Guide, External Interfaces, http://www.mathworks.com/help/techdoc/matlab_external/bp_kqh7.html, Acceso: 19 Enero (2012).

Pimpa C. y Premrudeepreechacharn S., Voltage Control In Power System Using Expert System Based On SCADA System, Proceedings of IEEE Power Engineering Society Winter Meeting: 2, $1282-1286$ (2002).

Saxena D., Singh S. N. y Verma K. S., Application of computational intelligence in emerging power systems, International Journal of Engineering, Science and Technology: 2(3), 1-7 (2010).

Thukaram D. y Yesuratnam G., "Fuzzy - Expert Approach for Voltage-Reactive Power Dispatch", Proceedings of Power Indian Conference, 199-206, New Delhi, India 10-12 April (2006).

Yesuratnam G. y Srilatha N., "An Expert System Approach of Congestion Management for Security and Economy Oriented Power System Operation", Proceedings of International Power and Energy Conference - IPEC 2010, 287-292, Singapore 27-29 October (2010).

Zimmerman R. D., Murillo-Sánchez C. E. y Gan D., MATPOWER A MATLAB Power System Simulation Package, Power Systems Engineering Research Center, http://www.pserc.cornell.edu//matpower/, Acceso: 17 Enero (2012). 
